"NOTICE: this is the author's version of a work that was accepted for publication in Biomedical Microdevices. Changes resulting from the publishing process, such as peer review, editing, corrections, structural formatting, and other quality control mechanisms may not be reflected in this document. Changes may have been made to this work since it was submitted for publication. A definitive version will be subsequently published in Biomedical Microdevices, [VOL.10(2), pp.153-67, (2008)].”

\title{
In vitro blood flow in a rectangular PDMS microchannel: experimental observations using a confocal micro-PIV system
}

\author{
Rui LIMA ${ }^{1,2}$, Shigeo WADA ${ }^{3}$, Shuji TANAKA ${ }^{4}$, Motohiro TAKEDA ${ }^{1,5}$, Takuji ISHIKAWA ${ }^{1}$, \\ Ken-ichi TSUBOTA ${ }^{1}$, Yohsuke IMAI ${ }^{1}$, Takami YAMAGUCHI ${ }^{1}$ \\ ${ }^{1}$ Department of Bioengineering and Robotics, Graduate School of Engineering, Tohoku University, 6-6-01 Aoba, \\ 980-8579 Sendai, Japan \\ ${ }^{2}$ Department of Mechanical Tech, ESTiG, Braganca Polyt., C. Sta. Apolonia, 5301-857 Braganca, Portugal \\ ${ }^{3}$ Department of Mechanical Science and Bioengineering, Graduate School of Engineering Science., Osaka \\ University, Toyonaka, 560-8531 Osaka, Japan \\ ${ }^{4}$ Department of Nanomechanics, Graduate School of Engineering, Tohoku University, 6-6-01 Aoba, 980-8579 \\ Sendai, Japan \\ ${ }^{5}$ Division of Surgical Oncology, Graduate School of Medicine, Tohoku University, 2-1 Seiryo-machi, Aoba-ku, \\ 980-8575 Sendai, Japan
}

E-mail: rui@pfsl.mech.tohoku.ac.jp

Key words: Microcirculation, confocal micro-PIV, PDMS microchannel, red blood cells, blood flow.

\begin{abstract}
Progress in microfabricated technologies has attracted the attention of researchers in several areas, including microcirculation. Microfluidic devices are expected to provide powerful tools not only to better understand the biophysical behavior of blood flow in microvessels, but also for disease diagnosis. Such microfluidic devices for biomedical applications must be compatible with state-of-the-art flow measuring techniques, such as confocal microparticle image velocimetry (PIV). This confocal system has the ability to (not only) quantify flow patterns inside microchannels with high spatial and temporal resolution, but can also be used to obtain velocity measurements for several optically sectioned images along the depth of the microchannel. In this study, we investigated the ability to obtain velocity measurements using physiological saline (PS) and in vitro blood in a rectangular polydimethysiloxane (PDMS) microchannel (300 $\mu \mathrm{m}$ wide, $45 \mu \mathrm{m}$ deep) using a confocal micro-PIV system. Applying this combination, measurements of trace particles seeded in the flow were performed for both fluids at a constant flow rate (Re $=0.02)$. Velocity profiles were acquired by successive measurements at different depth positions to obtain three-dimensional (3-D) information on the behavior of both fluid flows. Generally, the velocity profiles were found to be markedly blunt in the central region, mainly due to the low aspect ratio $(h / w=0.15)$ of the rectangular microchannel. Predictions using a theoretical model for the rectangular microchannel corresponded quite well with the experimental micro-PIV results for the PS fluid. However, for the in vitro blood with $20 \%$ haematocrit, small fluctuations were found in the velocity profiles. The present study clearly shows that confocal micro-PIV can be effectively integrated with a PDMS microchannel and used to obtain blood velocity profiles along the full depth of the microchannel because of its unique 3-D optical sectioning ability. Major advantages and disadvantages of PDMS microchannels over glass capillaries are also described.
\end{abstract}




\section{Introduction}

Blood is not a homogeneous fluid, but one composed mainly of a suspension of red blood cells (RBCs) in plasma. In addition, the non-continuum behavior of blood flow through microvessels leads to complex flow mechanics, which are not yet clearly understood (Goldsmith and Turitto 1986, Secomb 1995, Mchedlishvili \& Maeda 2001). Because several important physiological and pathological phenomena occur in the microcirculation, velocity profiles have been intensively studied both in vivo (Einav et al. 1975, Tangelder et al. 1986, Golster et al. 1999, Parthasarathi et al. 1999, Nakano et al. 2003) and in vitro (Gaehtgens et al. 1970, Baker \& Wayland 1974, Born et al. 1978, Cochrane et al. 1981, Gaehtgens 1987, Uijttewaal et al. 1994, Alonso et al. 1995, Moger et al. 2004, Kim \& Lee 2006, Lima et al. 2007) using several blood flow measuring techniques. Both in vivo and in vitro experiments have played important roles in our understanding of several phenomena in the microcirculation. Although several comprehensive reviews about blood flow behavior in microvessels have been published (Caro et al. 1978, Chien et al. 1984, Secomb 1995, Mchedlishvili \& Maeda 2001, Pries \& Secomb 2003), much remains unknown about the physiological and pathological phenomena occurring in the microcirculation.

Historically, the most commonly used experimental techniques to study blood flow in the microcirculation have been double-slit photometry (Gaehtgens et al. 1970, Baker \& Wayland 1974), video microscopy and image analysis (Bugliarello et al. 1963, Tangelder et al. 1986, Goldsmith and Turitto 1986, Gaehtgens 1987, Alonso et al. 1995, Parthasarathi et al. 1999, Tsukada 2000), and laserDoppler anemometry (Einav et al. 1975, Born et al. 1978, Cochrane et al. 1981, Uijttewaal et al. 1994, Golster et al. 1999). However, with these techniques, both spatial resolution and velocity accuracy are unsatisfactory.

In recent years, due to advances in computers, optics, and digital image processing techniques, it has become possible to combine a conventional particle image velocimetry (PIV) system (Adrian 1991, Raffael et al. 1998) with an inverted epifluorescent microscope (Santiago et al. 1998, Koutsiaris et al. 1999). As a result, this combination, known as a micro-PIV, has greatly increased the resolution of conventional PIV. Because of the success of the micro-PIV in measuring accurate velocity fields of homogeneous fluids in microfluidic devices (Nguyen \& Wereley 2002), the technique has been recently used to investigate blood flow behaviour in both microchannels and microvessels (Nakano et al. 2003, Bitsch et al. 2005, Vennemann P. et al. 2006).

Although the conventional PIV technique has proven useful in measuring blood flow velocities in microvessels, the entire flow field is illuminated and consequently the out-of-focus emitted light can result in high levels of background noise, which degrades the measured velocity fields (Meinhart et al. 2000, Nguyen \& Wereley 2002). One way to minimize the depth-of-focus effect is using a confocal micro-PIV (Tanaami et al. 2002, Kinoshita et al. 2005, Lima et al. 2005, 2006) or confocal laser scanning microscopy (CLSM) micro-PIV (Park et al. 2004, 2006). This technique combines the conventional PIV system with a spinning disk confocal microscope (SDCM), which has the ability to obtain in-focus images with an optical thickness less than $1 \mu \mathrm{m}$ (optical sectioning effect). Park et al. (2004, 2006) compared confocal micro-PIV with conventional micro-PIV and demonstrated that the former had improved particle image contrast and definition, allowing more accurate velocity measurements (see Table 1). As a result, by combining SDCM with a conventional PIV system, it is possible to achieve a PIV system with high spatial resolution able to generate velocity profiles at several optically sectioned planes along the microchannel depth.

Table 1. The lateral and axial resolutions of both conventional and confocal microscopes and optical slice thickness of confocal microscopes (Willhelm et al. 2003, Park et al. 2004).

\begin{tabular}{lcccc}
\hline \multicolumn{1}{c}{$\begin{array}{c}\text { Optical } \\
\text { resolutions }\end{array}$} & \multicolumn{2}{c}{$\begin{array}{c}\text { Conventional } \\
\text { microscopy }\end{array}$} & \multicolumn{2}{c}{ Confocal } \\
& $40 \times$ & $10 \times$ & $40 \times$ & $10 \times$ \\
\hline Lateral resolution $(\mu \mathrm{m})$ & 0.418 & 1.047 & 0.331 & 0.829 \\
Axial resolution $(\mu \mathrm{m})$ & 1.831 & 11.444 & 1.268 & 9.323 \\
Optical slice thickness $(\mu \mathrm{m})$ & \multicolumn{2}{c}{ Not definable } & 2.820 & 26.701 \\
\hline
\end{tabular}


Progress in microfabricated technologies has attracted the attention of the biomedical research community to the development of diagnostic, microfluidic devices (so-called bio-microelectromechanical systems or a lab-on-a-chip) (Sutton et al. 1997, Gomez et al. 2001, Beebe et al. 2002, Gifford et al. 2003, Minas et al. 2004, Toner and Irimia 2005, Faivre et al. 2006) capable of providing useful information about the general health status of patients. However, most of the information provided by blood analysis in microchannels is based on conventional visual observations on the area and volume of the RBCs (Sutton et al. 1997, Gifford et al. 2003). We believe that the acquisition of quantitative blood flow measurements may also provide relevant information for the diagnosis of disease and the treatment of patients.

Recently, we demonstrated the ability of the confocal micro-PIV to measure both pure water and diluted suspensions of blood cells in a glass square microchannel (Lima et al 2006); however, glass microchannels are not ideal to study the flow properties of blood at microscopic level mainly due to fabrication problems and physicochemicals factors (Maeda 1996). First, glass-to-glass sealing is difficult because direct bonding needs extremely smooth and flat surfaces, and indirect bonding fills the channels with adhesives, sols, and metals. Second, the fabrication of microchannels in glass is limited in depth and width, because glass is isotropically etched by hydrofluoric acid using metal mask, which has a limited durability against the etchant. Moreover, the most suitable objective lenses for the confocal micro-PIV system have limited working distances (up to $0.5 \mathrm{~mm}$ ), which requires special considerations in microchannel design. Besides the fabrication limitations, several physical and biochemical factors contribute to the flow behavior of blood cells through microvessels. Glass microchannels differ from microvessels in several ways: the wall is not elastic, the flow channel is often straight and long and the inner surface does not accommodate living endothelial cells. Hence, the results from classical in vitro experiments (Chien et al. 1984, Pries and Secomb 2003) that have used glass microchannels need to be carefully reexaminated by using more sophisticated microchannels, such as polydimethysiloxane (PDMS) microchannels.

Because of these limitations with glass microchannels, it is important to develop a microfluidic device closely representative of the in vivo microvascular environment using a reliable microfabrication technique compatible with the state-of-the-art flow measuring techniques, such as a confocal microPIV system. By using a soft lithography technique it is possible to generate extremely precise, reproducible and versatile rectangular microchannels. Although, rectangular microchannels may not be the best models to simulate in vivo microvessels geometry, many phenomena of blood flow behavior through this kind of microchannels exhibits certain features characteristic with those investigated in living microvessels (Beebe et al. 2002, Shevkoplyas et al. 2003, ,Toner and Irimia 2005, Faivre et al. 2006). Furthermore, experiments with this kind of microchannels enable not only precise control of experimental parameters but also obtain more accurate measurements. Thus, it is imperative to verify the applicability of the confocal micro-PIV to measure both physiological saline (PS) and in vitro blood with heamatocrits (Hct 20\%) close to the in vivo environment, in PDMS microchannels. To our knowledge confocal micro-PIV measurements of both PS and in vitro blood flow in a rectangular PDMS microchannel have not been published. Thus, as a first step towards integrating a confocal micro-PIV with a PDMS microchannel, measurements should be first performed in a simple straight rectangular microchannel.

In this study, we investigated the ability of our confocal micro-PIV system to measure the velocity profiles of physiological saline (PS) and in vitro blood at a normal Hct in a rectangular (300 $\mu \mathrm{m}$ wide, $45 \mu \mathrm{m}$ deep) PDMS microchannel, which was fabricated by soft lithography. In particular, we compare the behavior of PS and in vitro blood flow $(20 \% \mathrm{Hct})$ in a rectangular microchannel with a low aspect ratio. The results presented are also a verification of the applicability of the confocal microPIV to obtain detailed measurements of blood flow with Hcts similar to actual Hcts encountered in microvessels. 


\section{Materials \& Methods}

\subsection{Fabrication of the PDMS microchannel}

PDMS polymers have many useful properties, including good optical transparency and biocompatibility, easily reversible sealing to glass, elasticity, replication of fine and complex geometries, permeable to gases, thermally stable, and low cost (Duffy et al. 1998, Chang et al. 2003, Mata et al. 2005, Kaji et al. 2006). Because of these properties, this material was suitable for studying several phenomena in the microcirculation by combining it with our confocal micro-PIV system.

The PDMS rectangular microchannel was developed using a soft lithographic technique. A detailed description of the fabrication process can be found elsewhere (Lima 2007). In brief, the photomask was first created using a pattern generator (Nihon Seiko, Japan) and then a solid master was fabricated on a glass substrate with an ultrathick photoresist (SU-8 50; Kayaku MicroChem, Japan). Metal wire posts were placed on the SU-8 mold master to create the connection channels to the input/output ports. The PDMS prepolymer was prepared by mixing a commercial prepolymer and catalyzer (Silpot 184; Dow Corning, USA) at a weight ratio of 10:1. After the mixture was degassed under vacuum, the PDMS was poured into the mold master and cured by baking for about $2 \mathrm{~h}$ at $70^{\circ} \mathrm{C}$. Both master and PDMS were cooled to room temperature and the PDMS was peeled from the master. The embedded metal wire posts were then pulled out of the PDMS. Finally, the PDMS was washed with ethanol and brought into contact with a clean glass, where a reversible seal formed spontaneously. For this particular study we found no need to make the PDMS hydrophilic by using an oxygen plasma treatment.

The input/output ports were made by means of a $200 \mu \mathrm{l}$ micro-pipette tip. This tip was inserted tightly into the connection channels where it exerts pressure on the PDMS and provides a liquidproof seal. Top tubes (2.1 mm outer diameter , $1.1 \mathrm{~mm}$ inner diameter) were also fitted tightly into the micropipette in order to deliver the working fluids from the syringe pump (see Figure 1). Due to the conical shape of the tip and low flow rates we did not observe any fluid leakage during our experiments. Hence, we found no need to use additional bounding adhesives to avoid possible leakages from the microdevice.

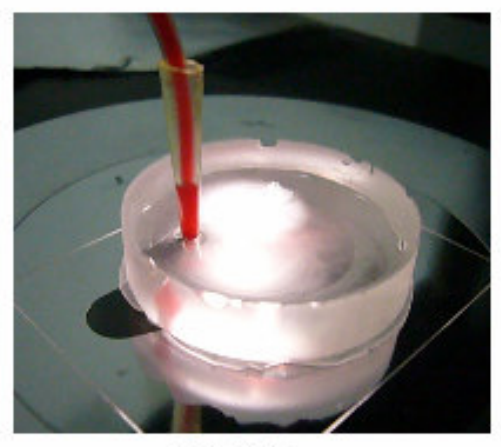

Top view

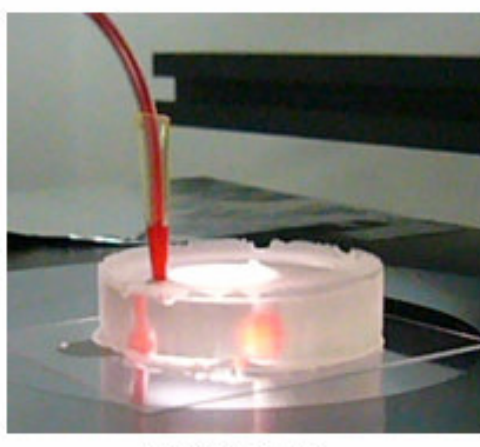

Lateral view

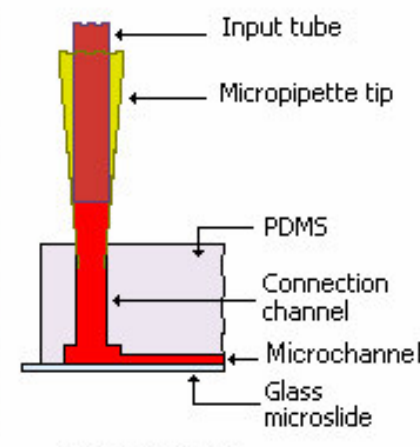

Cross section

Fig.1 PDMS microdevice with the input port (a) top view, (b) lateral view, (c) schematic drawing of the cross section.

To evaluate the performance of the confocal micro-PIV system by comparing experimental data with a theoretical model, it is important to both qualitatively and quantitatively verify the actual final geometry of the PDMS microchannel. Thus, the depth profile of the microchannel was carefully measured using a surface profiler (P-10; KLA Tencor, USA). Figure 2 shows the average depth from eight different measurements along the PDMS microchannel. The average depth was found to be $45 \mu \mathrm{m} \pm 1 \mu \mathrm{m}$. In addition, by using a wide-field microscope the average width was found to be $300 \mu \mathrm{m} \pm 2 \mu \mathrm{m}$. Therefore, the measurement uncertainties for the depth and width of the microchannel were $2.2 \%$ and $0.7 \%$, respectively. We also cut the PDMS microchannel transversely to measure the angle of the microchannel wall. We verified that the microchannel had a cross section that was nearly a perfect rectangular shape $(300 \mu \mathrm{m}$ wide, $45 \mu \mathrm{m}$ deep). Figure 3 shows the cross section of the rectangular PDMS microchannel under an optical microscope. 


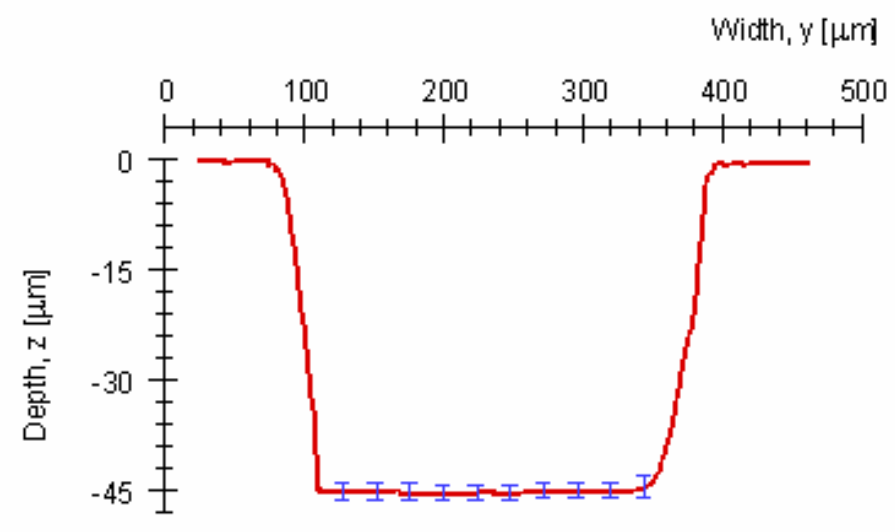

Figure 2. Average depth of the rectangular PDMS microchannel. The measured values of the depth are expressed as the means \pm standard deviation.

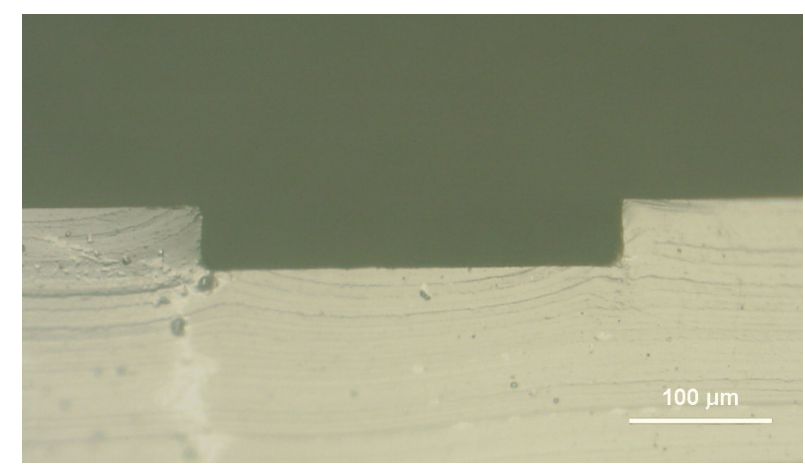

Figure 3. Cross section of the PDMS microchannel.

\subsection{Sample preparation: Working fluids and blood samples}

Two working fluids were used in this study: physiological saline (PS) and in vitro blood (PS containing $\sim 20 \%$ (by volume) human red blood cells (RBCs)). Fluids were seeded with $0.15 \%$ (v/v) $1-$ $\mu \mathrm{m}$-diameter red fluorescent solid polymer microspheres (R0100; Duke Scientific, USA). For the in vitro blood, the fluorescent particles were first washed twice before seeding into the blood cell suspension.

Venous blood ( $20 \mathrm{~mL}$ ) was collected from a healthy, adult volunteer (aged 32 years old) to make six samples with $\sim 20 \%$ (by volume) RBCs suspended in PS. To prevent coagulation of the blood cells during the experiment, ethylenediaminetetraacetic acid (EDTA) was added to the glass tube. The RBCs were separated from bulk blood by centrifugation (3000 rpm, $5 \mathrm{~min}$ ) and aspiration of the plasma and buffy coat, and were then washed twice with PS. The washed RBCs were diluted with PS to make up the required $\mathrm{RBC}$ concentration by volume. The haematocrit of the $\mathrm{RBC}$ suspension was about $20 \%(20 \% \mathrm{Hct})$ and the volume of each sample was $500 \mu \mathrm{L}$. Blood samples were stored at $4^{\circ} \mathrm{C}$ until the experiment was performed at room temperature $\left(25-27^{\circ} \mathrm{C}\right)$. All procedures in this experiment were carried out in compliance with the Ethics Committee on Clinical Investigation of Tohoku University.

\subsection{Confocal micro-PIV experimental setup}

The confocal micro-PIV system used in this study is shown in Figure 4. A detailed description of the confocal system can be found elsewhere (Lima et al. 2006). In brief, the confocal system consisted of an inverted microscope (IX71; Olympus, Japan) combined with a confocal scanning unit (CSU22; 
Yokogawa, Japan), a diode-pumped solid-state (DPSS) laser (Laser Quantum, UK) with an excitation wavelength of $532 \mathrm{~nm}$, and a high-speed camera (Phantom v7.1; Vision Research, USA). The laser beam was illuminated from the bottom of the microscope stage through a dry $20 \times$ objective lens with a numerical aperture (NA) of 0.75 . The light emitted from the fluorescent tracers flowing within the microchannel passed through a color filter into the scanning unit CSU22, where light was reflected by a dichromatic mirror onto a high-speed camera to record the PIV images.

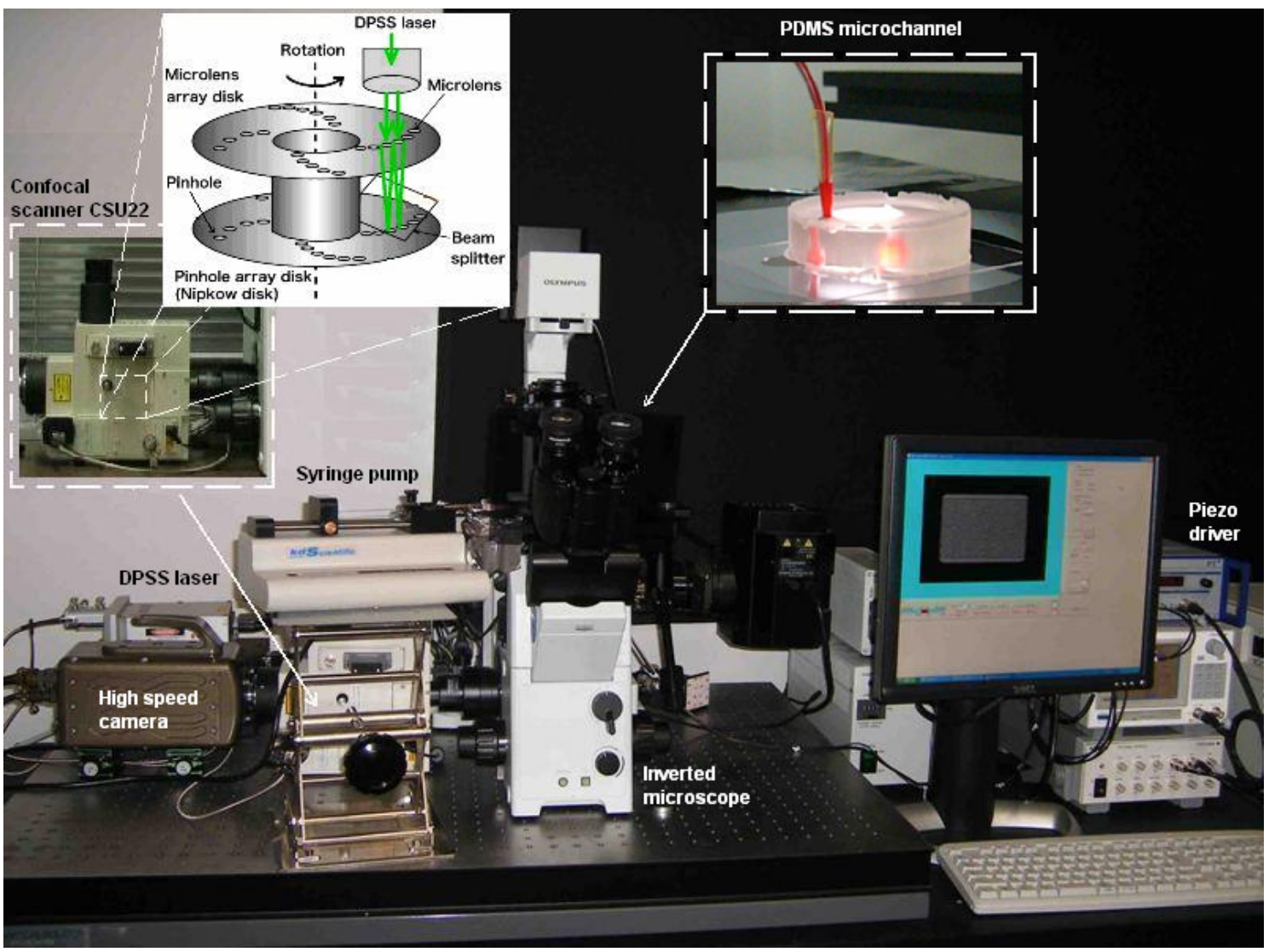

Figure 4. Confocal micro-PIV experimental setup.

The rectangular PDMS microchannel ( $300 \mu \mathrm{m}$ wide, $45 \mu \mathrm{m}$ deep, $10 \mathrm{~mm}$ long) was placed on the stage of an inverted microscope and using a syringe pump (KD Scientific, USA) a constant pressuredriven flow of $0.22 \mu \mathrm{L} / \mathrm{min}$ was maintained, corresponding to a Reynolds number ( $\operatorname{Re}$ ) of $\sim 0.02$ (based on the hydraulic diameter). A glass microsyringe ( $50 \mu \mathrm{L}$ capacity) was used to obtain a flow rate as close as possible to a steady flow.

For the working fluids, the PIV images were first captured with a resolution of $640 \times 480$ pixels, at a rate of $200 \mathrm{frames} / \mathrm{s}$ with an exposure time of $4,995 \mu \mathrm{s}$ and then recorded to a computer for evaluation, using the Phantom camera control software (PH607). Using a dry 20×/0.75 NA objective lens, the estimated thickness of the measurement plane (optical slice thickness) was $4.97 \mu \mathrm{m}$. Moreover, by using a high-speed objective lens actuator with submicron $z$-direction resolution it was possible to accurately capture optically sectioned images along the depth (z-axis) of the PDMS microchannel.

The PivView version 2.3 (PivTec, Germany) software (Willert et al. 1996, Rafael et al. 1998) was used to process the images by an iterative cross-correlation method (Willert et al. 1996, Rafael et al. 1998), while an advanced correlation technique (multi-grid interrogation) was used to improve the accuracy of the particle image displacement. The cross-correlation evaluation with grid refinement has been previously described (Willert et al. 1996, Rafael et al. 1998). In brief, the cross-correlation method is a statistical analysis that determines the average displacement by comparing the positions of 
the tracer particles within an interrogation area in two consecutive images. The cross-correlation function $\mathrm{R}_{\mathrm{fg}}(x, y)$ can be defined as (Rafael et al. 1998, Nguyen \& Wereley 2002):

$$
R_{f g}(x, y)=\sum_{i=1}^{p} \sum_{j=1}^{q} f(i, j) g(i+x, j+y),
$$

where $p$ and $q$ are the dimensions (in pixels) of the interrogation area, and $f(i, j)$ and $g(i, j)$ represent the grey value distribution of the first and second image, respectively. The cross-correlation function correlates images of particles in two consecutive interrogation areas (particle image pair). The displacement of a particle image pair corresponds to the highest peak in the correlation plane. However, when small interrogation areas (usually less then $32 \times 32$ pixels) are used, random correlations can result in noisy peaks and can generate an erroneous velocity vector (outlier). To reduce measurement uncertainty and also to improve the signal-to-noise ratio, a multi-grid interrogation technique can be applied to the cross-correlation analysis (Rafael et al. 1998). The evaluation procedure of this advanced correlation technique is first based on a cross-correlation analysis at a relatively high correlation area (usually $32 \times 32$ pixels or more) in order obtain an accurate estimation of the displacement and then the process is repeated at each pass by refining the interrogation area. By using this procedure the displacement at a smaller interrogation area can be estimated with high accuracy and consequently high-resolution velocity vectors can be generated.

In our study, we used the iterative cross-correlation method, in which a multi-grid interrogation algorithm (grid refinement) was selected for evaluating the PIV image pairs. The initial interrogation area was $64 \times 64$ pixels whereas the final size was $24 \times 32$ pixel (50\% overlapped) Furthermore, the least squares Gauss fitting algorithm was used for detecting the peak on the correlation plane. Finally, the data were converted to the corresponding velocity fields using a magnification factor of $0.86 \mathrm{pixel} / \mu \mathrm{m}$ and pulse delay of $5 \mathrm{~ms}$.

\subsection{Ensemble averaged velocities}

At steady flows, ensemble averaged velocities are known to improve the signal-to-noise ratio and consequently to generate more accurate velocity fields (Rafael et al. 1998, Meinhart et al. 2000). In this study, using the average velocity method, 100 instantaneous velocities measurements were ensemble-averaged to obtain the mean velocity field, $(\vec{U})$. This method first calculated the displacement field (peak detection) for each image pair and then the averaging operator was applied to all the instantaneous velocities measurements. The time-average mean velocity vector of the flow can be defined as

$\vec{U}=\frac{\sum_{t=1}^{n} u_{t}}{n}$,

where $n$ is the number of valid instantaneous velocity measurements. In this study, we evaluated 100 images with time intervals of $5 \mathrm{~ms}$.

By calculating the u-component of the time-average mean velocity, it is possible to obtain the timeaveraged velocity vector magnitude $(U)$, which can be expressed as

$U=|\vec{U}|$.

The time-averaged velocity vector magnitude for six different samples $(\bar{U})$ was defined as

$$
\bar{U}=\frac{1}{m \times n} \sum_{i=1}^{m} \sum_{i=1}^{n} U,
$$

where $m$ is the number of samples used and $n$ is the number of grid points along the $x$-direction. 


\section{Results}

\subsection{Physiological saline (PS)}

To evaluate the performance of the confocal micro-PIV system in measuring the velocity fields of the working fluids through the PDMS rectangular microchannel, the experimental results for PS were compared to a well established analytical solution for steady flow through a long, straight, rigid rectangular microchannel, that of Poiseuille flow. The equation used to calculate the analytical velocity profile of the rectangular microchannel with $y$ and $z$ cross section is the following (Bruus 2004, Mortensen et al. 2005):

$u_{x}(y, z)=\frac{48 Q}{\pi^{3} h w} \frac{\sum_{n, \text { odd }}^{\infty} \frac{1}{n^{3}}\left[1-\frac{\cosh \left(n \pi \frac{y}{h}\right)}{\cosh \left(n \pi \frac{w}{2 h}\right)}\right] \sin \left(n \pi \frac{z}{h}\right)}{\left[1-\sum_{n, \text { odd }}^{\infty} \frac{192 h}{n^{5} \pi^{5} w} \tanh \left(n \pi \frac{w}{2 h}\right)\right]}$,

where $u_{x}$ is the fluid velocity in the $x$-direction, $y$ and $z$ are the directions normal to the flow, $w$ and $h$ are the width and depth of the microchannel, respectively, and $Q$ is the flow rate.

Experimental PS $22.5 \mu \mathrm{m}$

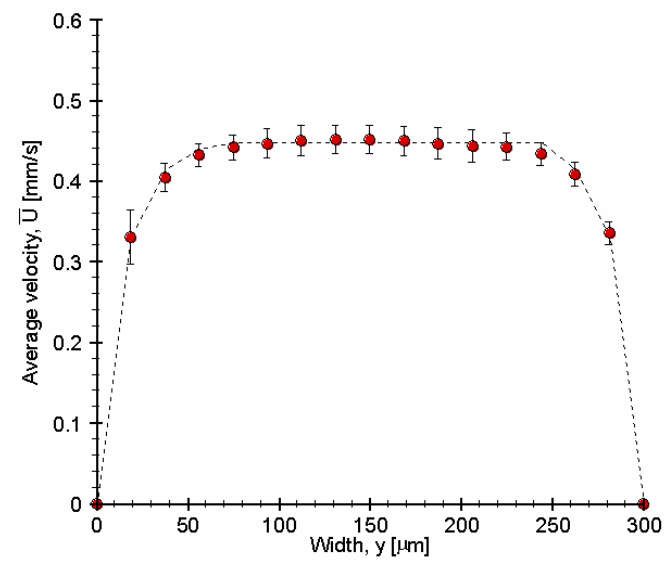

Figure 5. Comparison of experimental data and the theoretical model in the central plane $(22.5 \mu \mathrm{m})$. The error bars represent the standard deviation of six measurements using Student's t-test with a 95\% confidence interval.

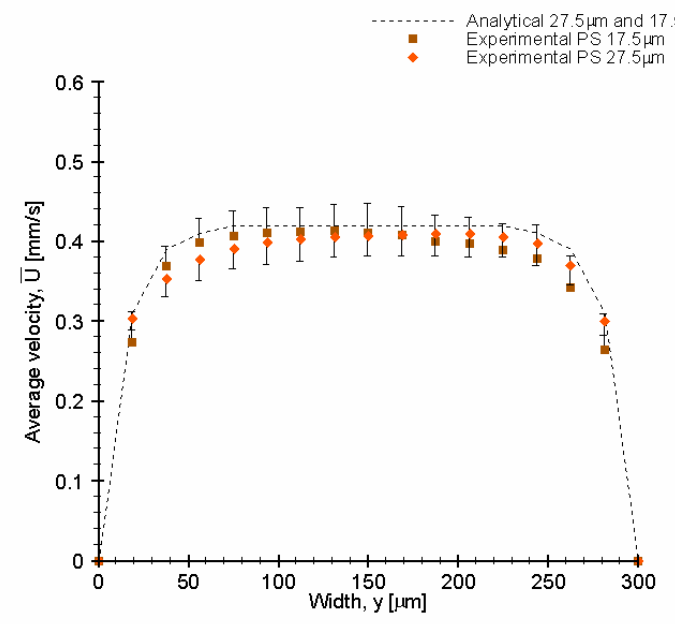

a)

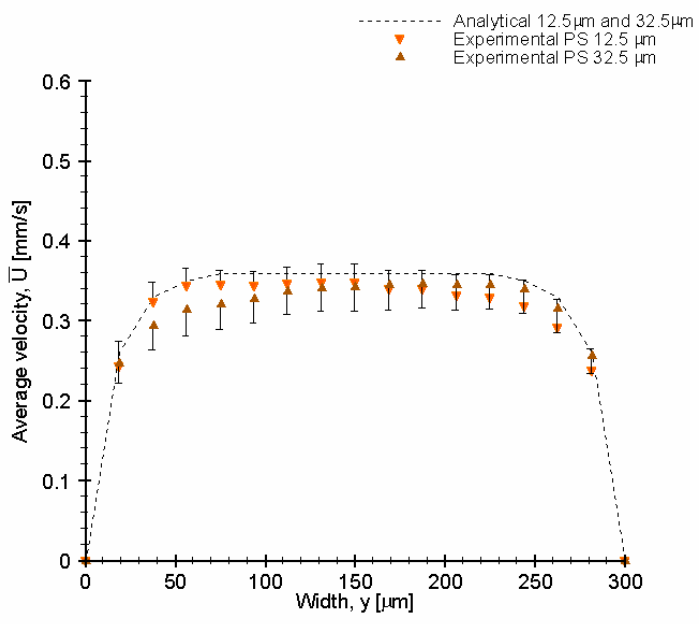

b)

Figure 6. Comparison of the experimental data and the theoretical model for optically sectioned images at (a) $z=17.5 \mu \mathrm{m}$ and $z=27.5 \mu \mathrm{m}$; (b) $z=12.5 \mu \mathrm{m}$ and $z=32.5 \mu \mathrm{m}$. 
Figures 5 and 6 compare the theoretical estimates using Equation 5 and the average fluid velocities of 100 PIV image pairs for several optically sectioned planes along the microchannel depth. The PIV measurements were obtained with an exposure time of $4995 \mu \mathrm{s}$, magnification factor (pixels $/ \mu \mathrm{m}$ ) of 0.86 , and time interval of $5 \mathrm{~ms}$ between two images.

Generally, the experimental results shown in Figures 5 and 6 were in good agreement with the analytical solution, especially the results at the middle plane $(\mathrm{z}=22.5 \mu \mathrm{m})$, where we found errors less than $5 \%$ (Fig. 5). As one moves out of the middle plane, the deviations start to increase and at locations close to the wall, the errors are around 7\% (Fig. 6).

\subsection{Blood flow}

3.2.1. Ensemble-averaged velocity profiles

In addition to examining PS, we studied the behavior of in vitro blood (RBCs in PS, 20\% Hct) through the rectangular PDMS microchannel. An example of a recorded image (halogen illumination source) of the in vitro blood flow is presented in Figure 7a. Figure $7 \mathrm{~b}$ displays an image with both RBCs (halogen illumination) and fluorescent particles (laser-emitted light). The latter image could be used to calculate the velocity fields of the in vitro blood flow.

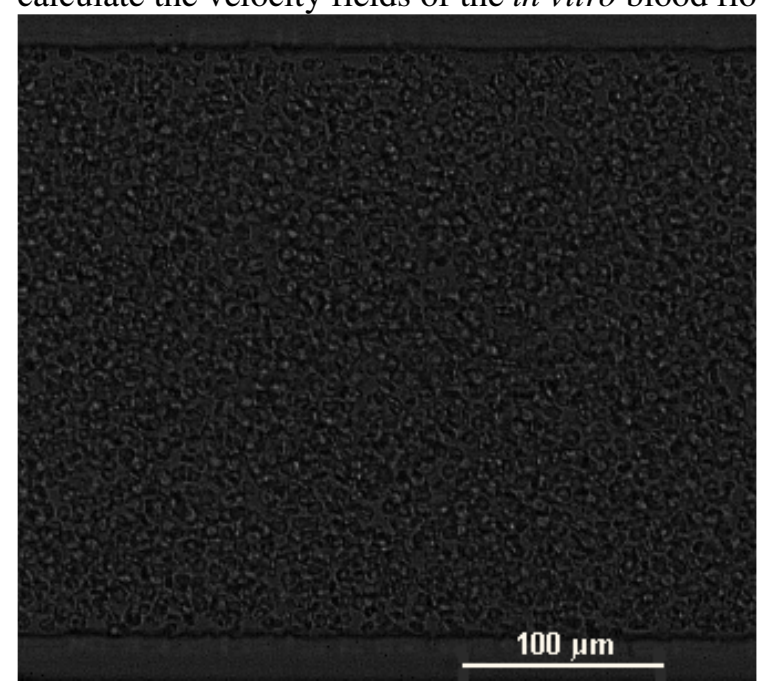

a)

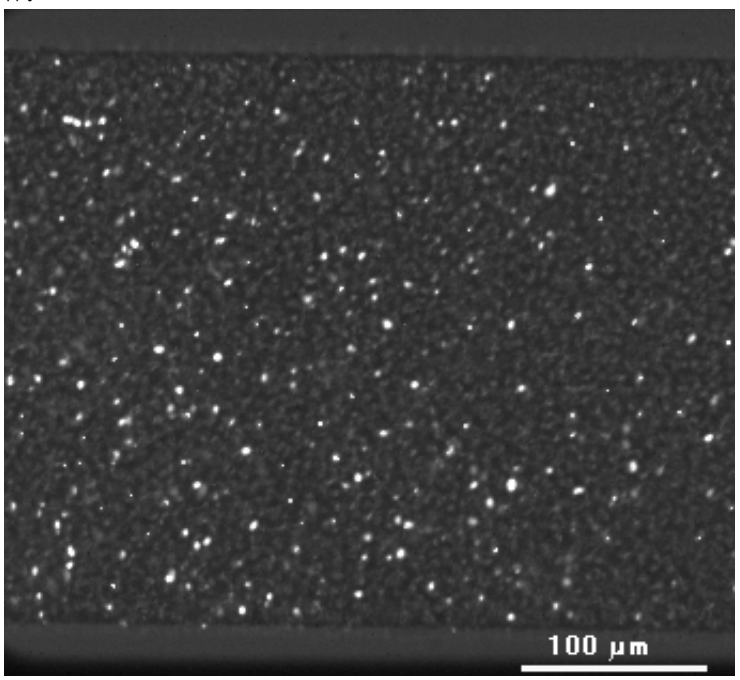

b)

Figure 7. (a) An image of in vitro blood (20\% Hct) flow with halogen illumination. The RBCs are observed as dark grey rings. (b) Image of the same fluid in which both fluorescent particles and RBCs are visualised.

Figures 8 and 9 compare the mean velocity profiles of 100 ensemble image pairs for PS and in vitro blood $(20 \% \mathrm{Hct})$, from six different measurements recorded at different $x-y$ planes, spaced at $5 \mu \mathrm{m}$ depth intervals ( $z$-direction). Both results were obtained under the same experimental conditions $(\mathrm{Re}=0.02)$. 


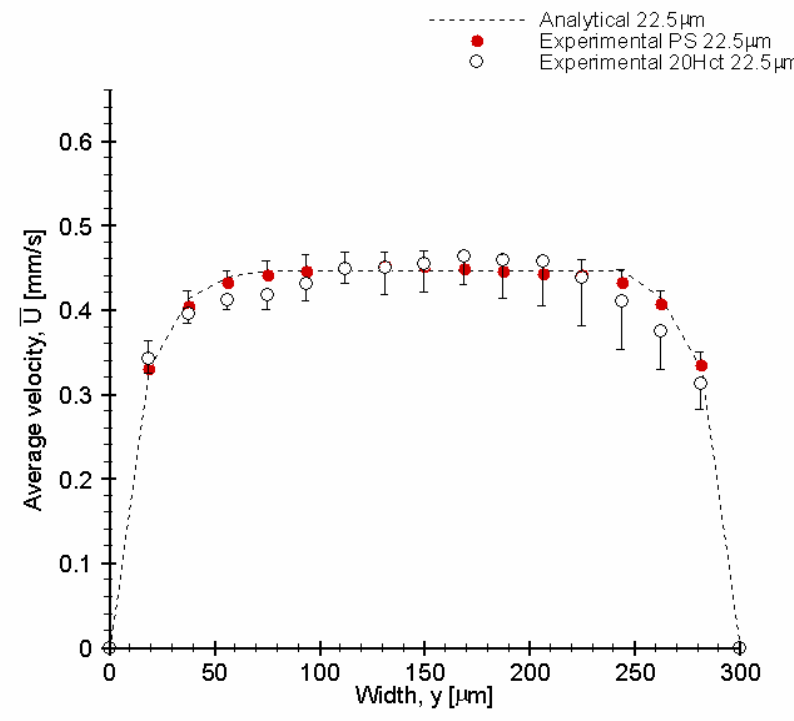

Figure 8. Comparison of ensemble-averaged velocity of PS and in vitro blood in the central plane $(22.5 \mu \mathrm{m})$.

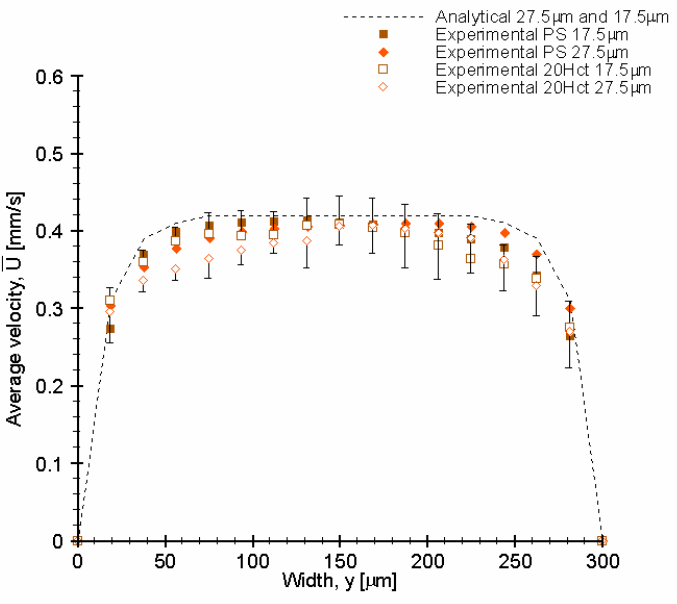

a)

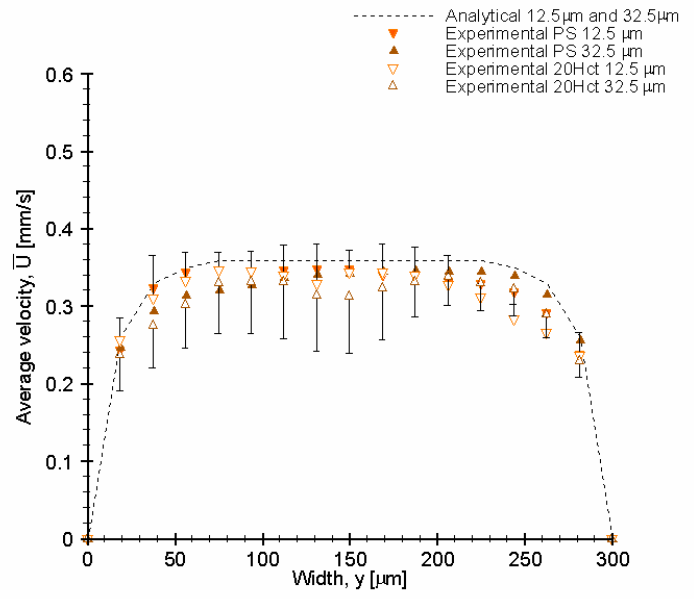

b)

Figure 9. Comparison of ensemble-averaged velocity of PS and in vitro blood at (a) $z=17.5 \mu \mathrm{m}$ and $z=27.5 \mu \mathrm{m}$; (b) $z=12.5 \mu \mathrm{m}$ and $z=32.5 \mu \mathrm{m}$.

From Figures 8 and 9, the velocity profiles of the PS fluid in several horizontal planes ( $x-y$ axis) clearly show that the flow is stable and fully developed. In addition, Figures 8 and 9 also show that for PS fluid, the shape of the mean velocity profile is markedly blunt in the middle region. However, examining the average velocity profiles of the in vitro blood, small deviations between the fluids were observed, especially in the regions near the walls. These small perturbations along the width of the microchannel become much clearer on analyzing the 3-D profiles shown in Figure 10. From Figure 10a indicates that the PS velocity profile along the $z$-direction had a smooth parabolic shape, whereas the in vitro blood flow (Fig. 10b) was characterized by a velocity profile containing several small disturbances along the $z$-direction. 


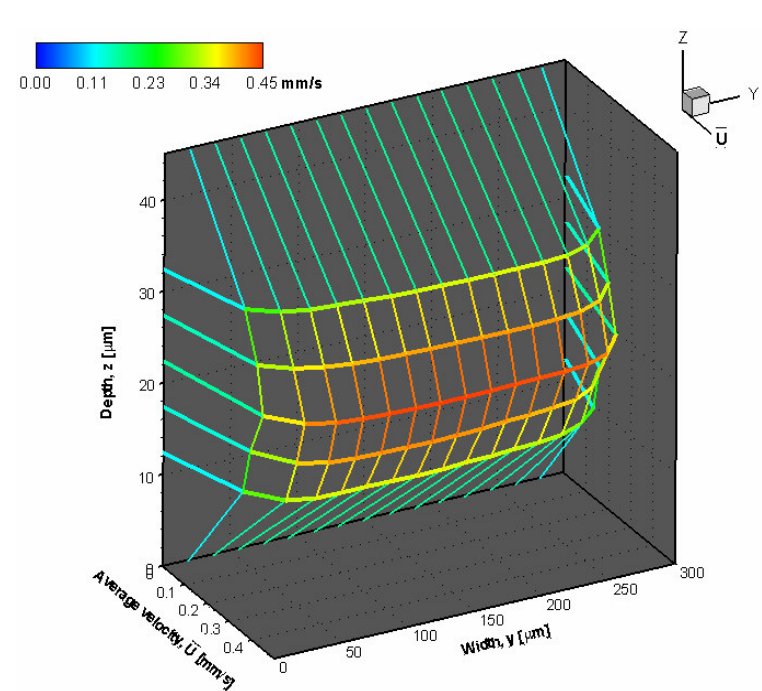

a)

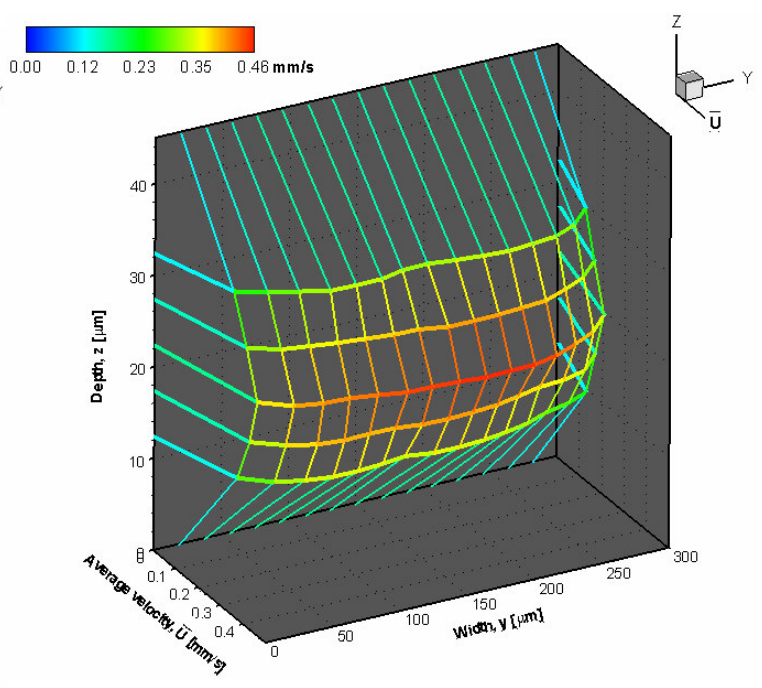

b)

Figure 10. Three-dimensional representation of optical sectioning velocity profiles of (a) PS and (b) in vitro blood.

\subsubsection{Ensemble velocity profiles in the middle plane}

It is generally agreed that the ensemble average velocity process improves the reliability and accuracy of PIV measurements because of the improvement in the signal-to-noise ratio and the reduction of measurement errors associated with the Brownian motion of the tracer particles and some possible "bottleneck" effects of the syringe pump. Furthermore, our experimental confocal PIV results with the PS fluid compared to the theoretical model (Fig. 5) showed the best agreement in the middle plane of the microchannel $(z=22.5 \mu \mathrm{m})$ because of a reduction in several effects resulting from the microchannel wall (interaction between particle and wall, roughness of the wall, etc.). The PIV measurements in the middle plane seemed to generate results closer to the true velocity profile present in the rectangular PDMS microchannel. In this study, six samples of 100 PIV image pairs at the middle plane were analyzed for both PS and in vitro blood $(\sim 20 \% \mathrm{Hct})$. Figures 11 and 12 show the time-averaged velocity profiles $(U)$ along a certain length $(150 \mu \mathrm{m})$ from two different representative samples for PS and in vitro blood, respectively.

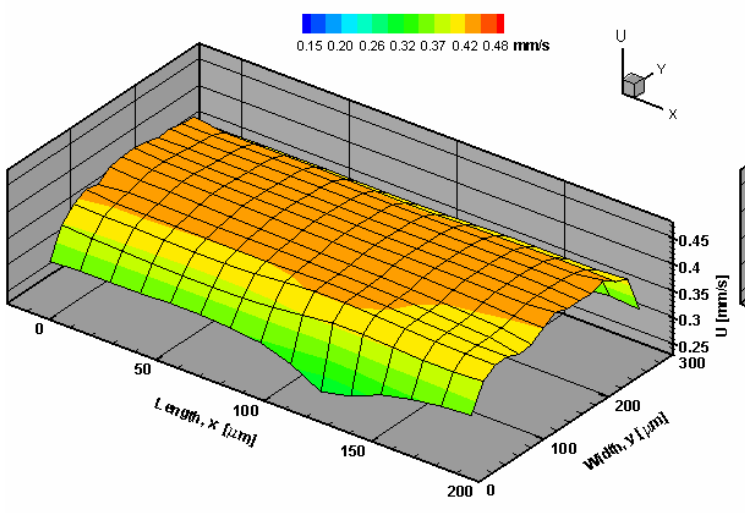

a) sample 1

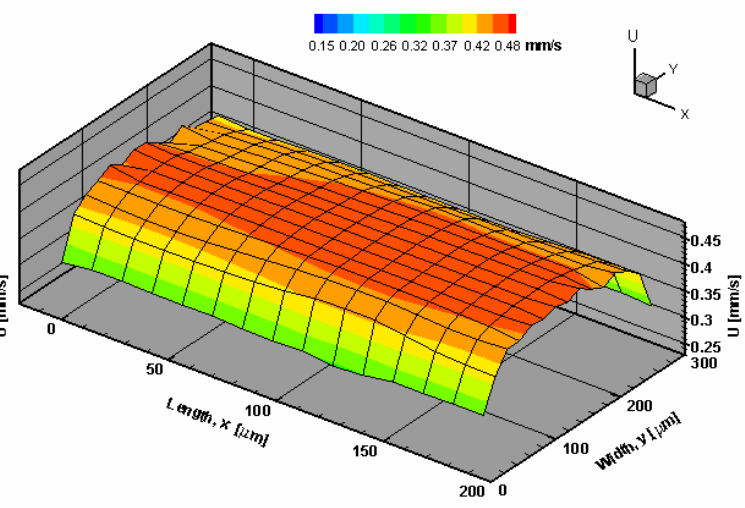

b) sample 2

Figure 11. Ensemble velocity profiles (U) of two representative samples in the middle plane for PS 


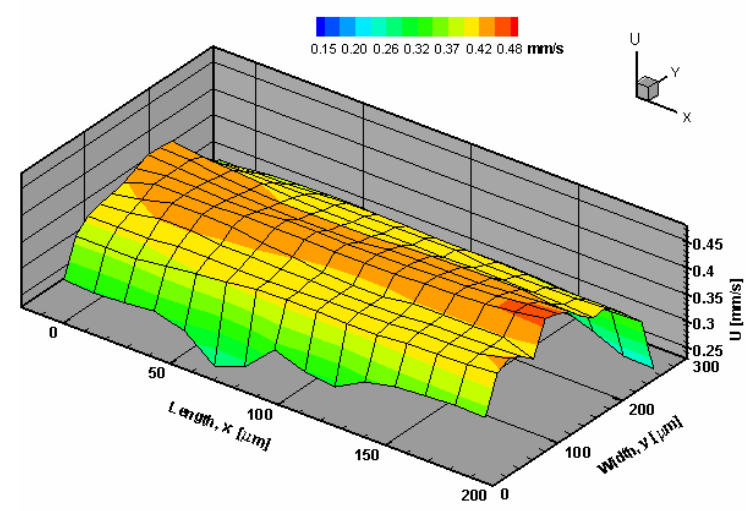

a) sample 1

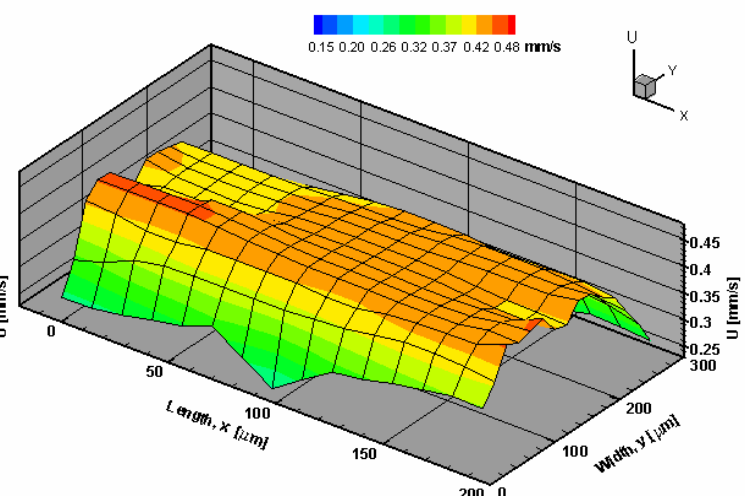

b) sample 2

Figure 12. Ensemble velocity profiles (U) of two representative samples in the middle plane for in vitro blood.

Figure 12 shows that when using PS fluid, a markedly smooth, flat profile tended to develop along the rectangular microchannel. However, for the in vitro blood flow, small perturbations in the shape of the ensemble velocity profiles were observed (Fig. 12). These qualitative observations can play an important role in providing reliable information on the time-averaged flow behavior over a specific period of interest.

\subsubsection{RBC flow visualization}

To determine possible causes of the perturbations in the velocity profiles of the in vitro blood flowing in the rectangular microchannel, RBC motion was visualized with halogen illumination from our confocal micro-PIV system. Figure 13 shows the dynamic behavior of the RBCs in a rectangular microchannel with a low aspect ratio. Figure 13 also shows detailed consecutive halogen images captured at time intervals of $10 \mathrm{~ms}$, when the motion of one particular RBC was followed.
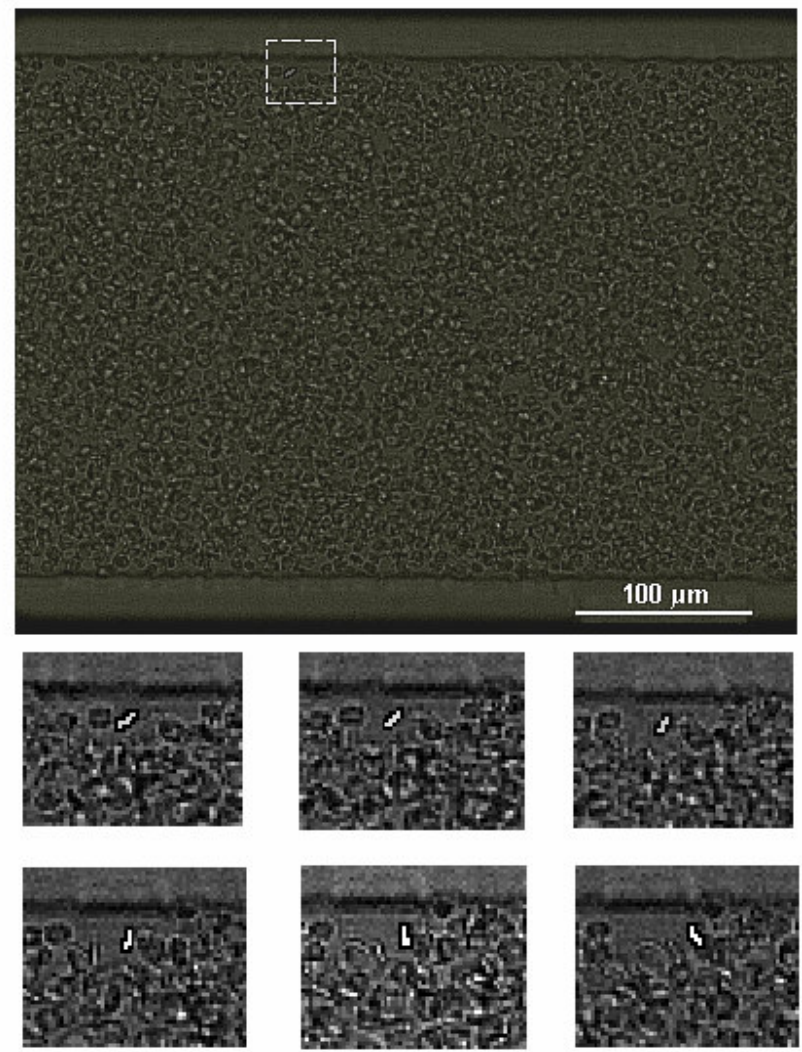

Figure 13. Motion of one RBC flowing in a rectangular microchannel with a low aspect ratio $(\mathrm{h} / \mathrm{w}=0.15)$. The $\mathrm{RBC}$ of interest has a bright grey colour in its central region. All images were captured with a $20 \times$ objective lens. 
During the capture of the images, we did not observe any significant plasma layer, but rather a homogenous distribution of the RBCs. Furthermore, in the central region of the microchannel (shear rate close to zero), the RBCs tended to move along with the flow with a regular rotational motion, retaining their biconcave shape. Near to the wall, where the shear rate increases significantly, both rotational and tumbling motions accompanied by membrane deformation were observed (see detailed consecutive images in Fig. 13). Interactions between neighboring RBCs at different depth planes (boundary layers) were also observed along the full microchannel.

\subsubsection{Plasma layer}

The formation of a plasma-rich layer located between the RBC core and wall is well documented in the literature on glass microchannels with diameters less than $300 \mu \mathrm{m}$ (Faharaeus and Lindqvist 1931, Chien et al. 1984, Maeda 1996, Pries and Secomb 2003). However, to our knowledge the effect of both geometry and suspension medium in a straight rectangular PDMS microchannel has not been investigated. In an effort to understand the causes for no observation of any plasma layer in our microchannel, firstly we have investigated the effect of the flow rate on the formation of the plasma layer. Figure 14 shows in vitro blood ( $20 \%$ Hct in PS suspension medium) through the rectangular PDMS microchannel, for two different flow rates. The results illustrate that the RBCs flowing in this particular microchannel ( $300 \mu \mathrm{m}$ wide, $45 \mu \mathrm{m}$ deep) do not have tendency to migrate toward the centre axis for Re up to 0.1 .

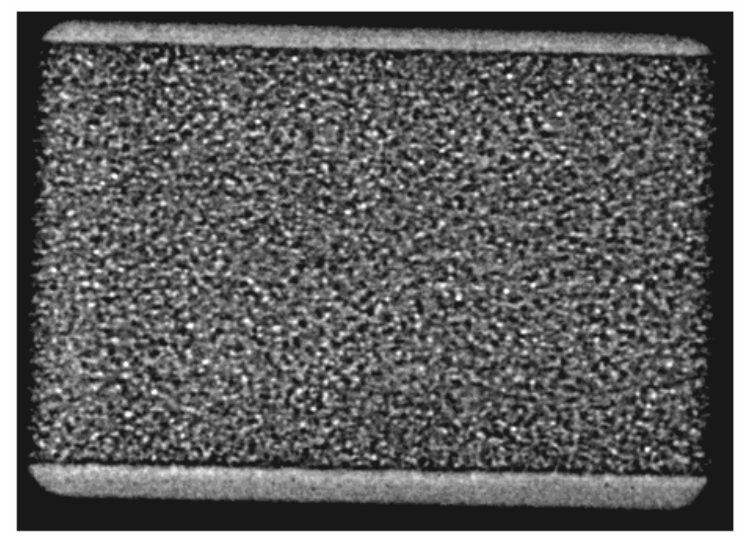

a)

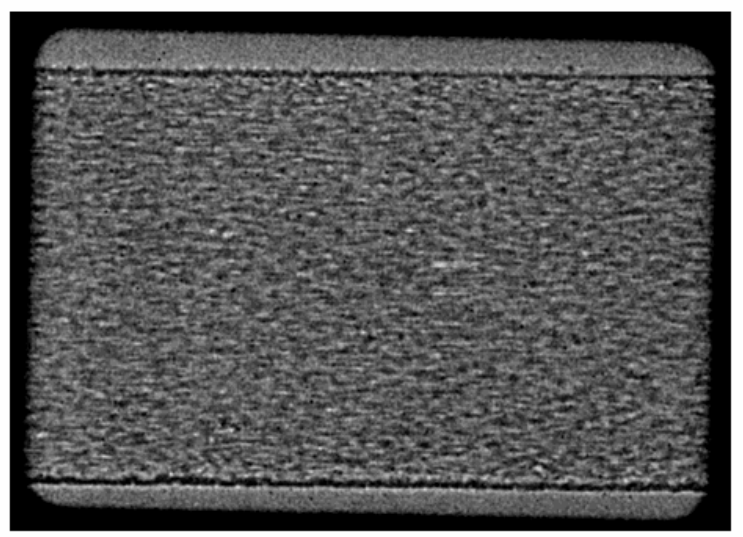

b)

Figure 14. Flow of RBCs ( 20\% Hct) suspended in PS through a rectangular PDMS microchannel $300 \mu \mathrm{m}$ wide and $45 \mu \mathrm{m}$ deep for (a) $\operatorname{Re} \sim 0.02$ (b) $\operatorname{Re} \sim 0.1$.

We next investigated the effect of the suspension fluid. In Figure 15 we show human RBCs $(\sim 20 \%$ Hct) suspended in dextran 40 (Dx-40, Otsuka Medicine) for Re up to 0.08. By using this high molecular suspension fluid, we eliminate the effects of sedimentation of the RBCs at low-shear rates. The qualitative results from Figure 15 show also no tendency for the RBCs to undergo axial migration.

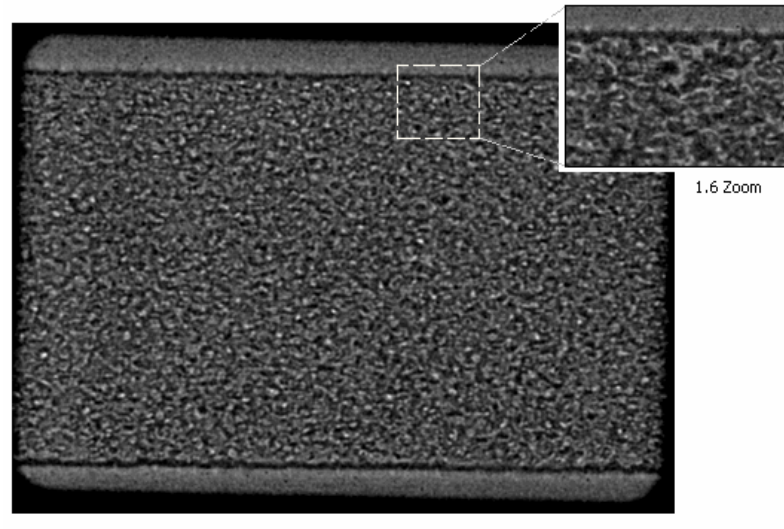

a)

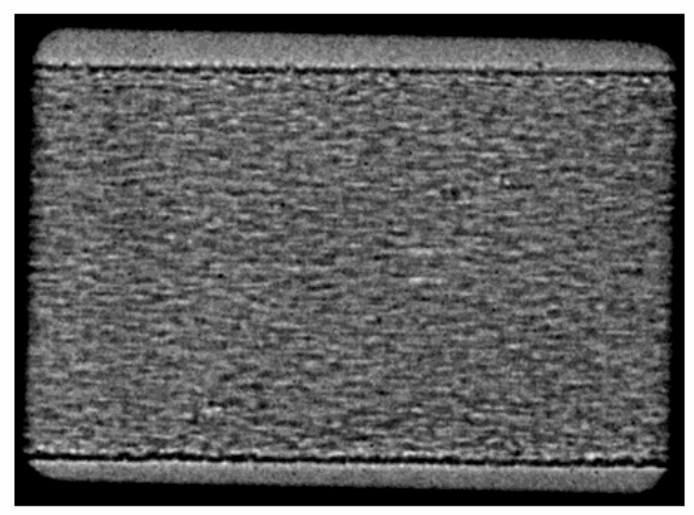

b)

Figure 15 Flow of RBCs ( 20\% Hct) suspended in dextran 40 (Dx-40) through a rectangular PDMS microchannel $300 \mu \mathrm{m}$ wide and $45 \mu \mathrm{m}$ deep for (a) $\operatorname{Re} \sim 0.01$ (b) $\operatorname{Re} \sim 0.08$. 
Finally, we investigated the flow behavior of RBCs ( $20 \% \mathrm{Hct})$ suspended in Dx-40 through a smaller PDMS microchannel $(100 \mu \mathrm{m}$ wide, $62 \mu \mathrm{m}$ deep), for a $\operatorname{Re} \sim 0.03$ (see Figure 16). In contrast to the previous results, the RBCs flowing through this PDMS microchannel have tendency to migrate toward the microchannel axis, promoting the formation of a plasma layer. Although we do not have extensive data on the influence of the width, it is nevertheless clear that the formation of the plasma layer is enhanced as the width becomes narrower.

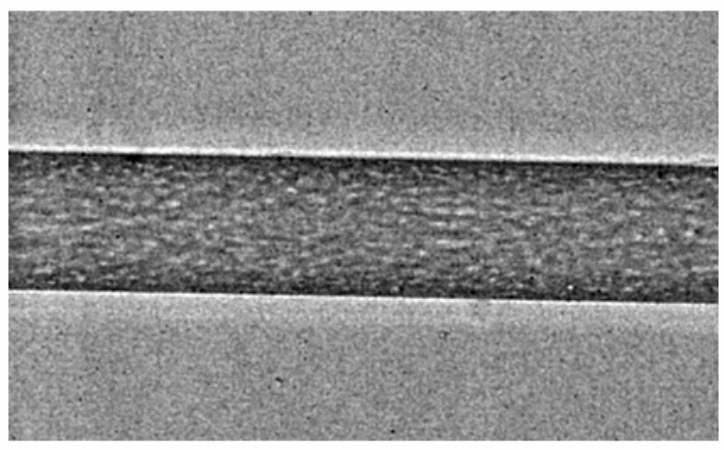

a)

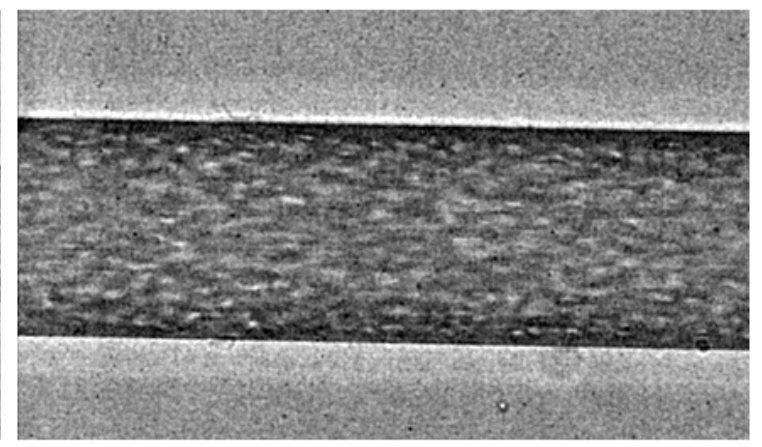

b)

Figure 16. Flow of RBCs ( 20\% Hct) suspended in dextran 40 (Dx-40) through a rectangular PDMS microchannel $100 \mu \mathrm{m}$ wide and $62 \mu \mathrm{m}$ deep for $\operatorname{Re} \sim 0.03$ (a) $20 \times$ objective lens (b) $32 \times$ objective lens.

\section{Discussion}

\subsection{Flow behavior of PS and in vitro blood through a PDMS microchannel}

In the present study, we determined the velocity profiles of PS fluid and in vitro blood (20\% Hct) in a rectangular PDMS microchannel with a low aspect ratio $(\mathrm{h} / \mathrm{w}=0.15)$ using a confocal micro-PIV system. In the case of PS fluid, our results were in good agreement with the analytical solution around the central plane $(z=17.5 \mu \mathrm{m}, z=22.5 \mu \mathrm{m}$, and $z=27.5 \mu \mathrm{m})$. However, at locations closer to the wall, the errors were slightly larger than $7 \%$. We believe that these larger errors were primarily attributable to "second-order effects," such as the surface roughness of the wall and background noise generated from particles adhering on the wall (bias effect). Although it is important to consider the data from all planes, we believe that the best results from the confocal system were presented in the middle plane. These results also demonstrated that around the central region, PS velocity profiles were markedly blunt, which is consistent with the characteristic behavior of a Newtonian fluid through a rectangular microchannel with a low aspect ratio.

By comparing the averaged ensemble velocity profiles of PS fluid and in vitro blood flow, we observed small deviations at all optically sectioned planes. These deviations were found to be larger at locations closer to the wall. Furthermore, the ensemble velocity profiles at the central plane for the in vitro blood were not characterized by a flat profile in the central region; rather, the profile had small perturbations. The reasons for these small perturbations are still not completely understood. However, from the visualization of the RBCs' motion through the rectangular microchannel, interactions between neighboring RBCs seem to be an important factor to take into account. The abrupt increase in the shear rate in the vicinity of the wall likely plays an important role in the local disturbance effects caused by both rotational and tumbling motion of deformable RBCs within the fluid flow. Moreover, temporal variation in the haematocrit could be another factor contributing to the perturbations described above. From the six measurements (Fig. 12 shows two of six) performed at the centre plane at different time periods, all show random perturbations on the averaged ensemble velocity profiles. This suggests that the transverse motion of the RBCs fluctuates over time, consequently affecting the local apparent viscosity of the fluid. Effects such as Brownian motion, microelectrohydrodynamics, and bottlenecks (Tabeling 2001, Nguyen \& Wereley 2002) seem unlikely to contribute significantly to the perturbations found in the in vitro blood velocity profiles because such perturbations were small when compared to the PS fluid profiles measured under the same experimental conditions. The present results suggest that the presence of deformable RBCs suspended within the plasma flow plays an important role on the hydrodynamic disturbance effect seen on the in vitro blood velocity profiles. Note that, these observations are consistent with several other measurements performed in glass microchannels (Goldsmith and Turitto 1986, Alonso et al. 1995, Lima et al. 2007). Further studies to 
clarify the presence of these fluctuations on the ensemble velocity profiles need to be conducted, not only using microchannels which closely mimic the actual human microvessels, but also using higher resolution objective lenses, such as $40 \times$ and $60 \times$.

\subsection{Plasma layer in a straight PDMS microchannel}

It is well known that RBCs, in microvessels less than $300 \mu \mathrm{m}$ (Faharaeus and Lindqvist 1931, Chien et al. 1984, Pries and Secomb 2003), tend to migrate towards the axis promoting the formation of a plasma layer adjacent to the vascular walls. In the present study we have used a PDMS microchannel with a width of $300 \mu \mathrm{m}$ and depth $45 \mu \mathrm{m}$. For this particular microchannel no plasma layer was observed during our experiments. In order to understand the formation of the plasma layer in PDMS microchannels we have investigated the effect of different parameters on the formation of the plasma layer, including geometry, flow rate and suspending media. Under similar conditions, our results have showed that for a $300 \mu \mathrm{m}$ wide microchannel no plasma layer was formed whereas for $100 \mu \mathrm{m}$ width a thin plasma layer was clearly observed. Thus, our qualitative results indicate that the geometry of PDMS microchannel is the main responsible for the formation of the plasma layer. In addition, our results also indicate that the plasma layer is enhanced as the width becomes narrower. This finding is consistent with a recent work performed on the flow of RBCs in model constrictions (Faivre et al. 2006).

\subsection{PDMS versus glass microchannels}

Several studies on blood flow in glass microchannels and in microvessels have yielded conflicting results with respect to flow resistance and deformability of RBCs (Pries at al. 1994, Suzuki et al. 1996). However, the observed in vivo/in vitro discrepancies have not yet been convincingly explained by using the research done in straight rigid glass microchannels. One possible way to overcome the limitations of the glass capillaries is by using PDMS microchannels. PDMS microchannels differ from glass capillaries in several ways (see also Table 2):

1. the permeability to gases, such as oxygen, has the unique advantage to culture living endothelial cells near to confluence (Borenstein et al. 2002, Shin et al. 2004) in closed microchannels, a task extremely difficult to achieve in glass capillaries. Very recently, a cellular micropatterning technique based on electrochemical method (Kaji et al., 2006) has also successfully cultured endothelial cells on the surfaces of PDMS microchannel;

2. PDMS is an elastomeric and biocompatible material. The typical ultimate tensile stress (UTS) for PDMS ranges from 3.9 MPa to 10.8 MPa (Mata et al. 2005), whereas the Young's elastic modulus (E) has a value of $\sim 0.75 \mathrm{MPa}$ (Unger et al. 2000). The UTS and $\mathrm{E}$ of the blood vessels has values from 0.4 MPa to 1.4 MPa (Holzapfel et al 2005) and 2.5 MPa (Steiger et al. 1989) respectively. The UTS and E is PDMS formulation dependent, by adjusting the ratio of the crosslinker (curing agent) and also the thickness of the wall, PDMS may be able to mimic more closely the geometric and structural properties of in vivo microvessels;

3. easy to replicate complex microvascular networks with submicron fidelity (McDonald et al. 2002, Shevkoplyas et al. 2003, Shin et al. 2004, Lima 2007);

4. due to its spontaneous adhesion onto glass substrates, PDMS sealing does not need any complex bonding technique. Hence, PDMS microchannels besides offering optimum optical access for confocal systems, they can be reused several times because of its reversible bonding. Glass-to-glass sealing requires elaborate bonding processes such as direct, thermal compression or hydrofluoric bonding method, which are time consuming and need very careful processing to obtain an acceptable yield. Using bonding interlayers such as silicon for anodic bonding, metal for fusion or eutectic bonding and spin-on-glass for thermal compression bonding are other options, however the interlayer are also deposited in the microchannel. In addition, all of these bonding methods need flat, smooth and clean surfaces, which requires a clean room facility.

5. possible to integrate into the PDMS device several functional components such as heaters, micromixers, micropumps, microvalves, temperature and pressure sensors, etc (Thorsen et al. 2002, Fujii 2002).

6. no need to use a refractive-index-matching liquid to minimize refraction from the walls. As a result, it is possible to use high magnification objective lenses $(40 \times, 60 \times, 100 \times)$ with high NA, which have typically working distance from 100 to $200 \mu \mathrm{m}$. Consequently it is possible to obtain images with much higher resolution. 
Table 2. Major advantages of PDMS microchannels over glass capillaries.

\begin{tabular}{|c|c|c|c|}
\hline $\begin{array}{c}\text { Physicochemical factors } \\
\text { that affects blood flow } \\
\text { behavior }\end{array}$ & $\begin{array}{c}\text { Glass } \\
\text { capillaries }\end{array}$ & $\begin{array}{c}\text { PDMS } \\
\text { microchannels }\end{array}$ & Comments \\
\hline $\begin{array}{l}\text { Ability to culture } \\
\text { endothelial cells near } \\
\text { to confluence }\end{array}$ & $\begin{array}{l}\text { complex in closed } \\
\text { microchannels }\end{array}$ & possible & $\begin{array}{l}\text { Recently techniques have been } \\
\text { developed to culture endothelial cells } \\
\text { near to confluence in PDMS } \\
\text { microchannels } 1,2,3 \text {. To the best of } \\
\text { our knowledge, no work has reported } \\
\text { culture cells near to confluence in } \\
\text { closed glass microchanels. This is } \\
\text { only possible by using parallel plate } \\
\text { flow chambers }{ }^{4} \text {. }\end{array}$ \\
\hline
\end{tabular}

Mechanical properties, ultimate tensile stress (UTS)

Young's elastic modulus (E) rigid material

(UTS $\sim 300 \mathrm{MPa}$ to $900 \mathrm{MPa})^{5}$ $(\mathrm{E} \sim 70 \mathrm{GPa})^{5}$

flexible material

(UTS $\sim 3.9 \mathrm{MPa}$ to $10.8 \mathrm{MPa})^{6}$ $(\mathrm{E} \sim 0.75 \mathrm{MPa})^{7}$

PDMS microchannels may be able to mimic the deformability of in vivo microvessels (UTS $~ 0.4 \mathrm{MPa}$ to 1.4 $\left.\mathrm{MPa}^{8}, \mathrm{E} \sim 2.5 \mathrm{MPa}^{9}\right)$. PDMS allows large deflections with small actuation forces ${ }^{7}$.

Presently it is possible to fabricate rectangular PDMS microchannels with complex geometries ${ }^{10,11}$.

Fabrication of microchannels in PDMS is simple, rapid and has high replication fidelity. In contrast, fabrication in glass increases the time, complexity, and cost of the fabrication process ${ }^{12}$.

References : ${ }^{1}$ Borenstein et al. 2002, ${ }^{2}$ Shin et al. 2004, ${ }^{3}$ Kaji et al. 2006, ${ }^{4}$ Brown 2000, ${ }^{5}$ Rika nenpyo 1996, ${ }^{6}$ Mata et al. 2005, ${ }^{7}$ Unger et al. 2000, ${ }^{8}$ Holzapfel et al. 2005, ${ }^{9}$ Steiger et al. 1989, ${ }^{10}$ Shevkoplyas et al. 2003, ${ }^{11}$ Lima 2007; ${ }^{12}$ McDonal et al. 2002.

In addition, by using a soft lithography technique it is possible to fabricate easily precise and reproducible rectangular microchannels at low cost, because the master mold is reusable. Rectangular PDMS microchannel with a low aspect ratio seems to be appropriate to perform confocal micro-PIV measurements of in vitro blood with Hcts close the actual in vivo Hct in microcirculation. However, this geometry may not reflect the actual physiology of the microcirculation. Hence, very recently we have successfully fabricated straight circular PDMS microchannels by using a wire casting technique (Lima, 2007). The fabrication of circular PDMS microchannels with complex geometries still remains a big challenge. Recently, Borenstein and his co-workers $(2002,2004)$ are developing a precise alignment technique to produce true cylindrical channels. However, the complexity of the alignment procedure, time-consuming and the thickness of the two matching PDMS templates containing the semicircular microchannels, may limit its practical applications onto confocal systems.

Nevertheless the limitations of PDMS microchannels, it is clear that this kind of microdevices exhibit features more close to the actual physiological conditions of the in vivo microvessels when compared with glass capillaries. Although PDMS microdevices are promising to mimic the in vivo microvascular environment, many challenges remain. First, the current PDMS fabrication processes need to be improved in order to produce precise circular microchanels with thin walls less than $200 \mu \mathrm{m}$ to obtain accurate measurements by means of confocal systems. Second, PDMS collapsible microchannels need to be developed in order to mimic the deformability of blood vessels more closely. Finally, it is also imperative to evaluate if the current cell culture techniques used in PDMS microchannels create a microvascular environment closely representative of the in vivo situation. 


\subsection{Ongoing development}

The in vitro research performed with classical glass microchannels has revealed several astonishing phenomena happening in microcirculation. Some examples are the Fahraeus effect and FahraeusLindqvist effect (Chien et al. 1984). However, recently several studies on flow resistance (Pries at al. 1994) and RBCs deformation (Suzuki et al. 1996) have shown quantitative difference between the results in microvessels and in glass microchannels. The reason for these differences still remains unclear mainly because glass microchannels differ from microvessels in several aspects, such as elasticity and geometry of the microchannel and biological effect by the endothelial inner surface. We believe that these limitations encountered in glass microchannels can be overcome by using a PDMS microchannel (see Table 2). Nevertheless, before moving to a more elaborated PDMS microchannel, we must first understand the flow behavior of blood in simple geometry similar to the one used in the present study. To fully exploit the potential of this combined system, we are working to develop a synergetic biochip to mimic the in vivo environment. By using an innovative cellular micropatterning technique based on electrochemical method (Kaji et al., 2006), we expect to culture endothelial cells on the surfaces of the PDMS microchannel to near confluence. In addition, by combining this microdevice with the confocal system, we will be able to identify and quantitatively measure cellular and molecular events occurring between the blood cells and endothelium. As a longer range goal, we believe that this ongoing research will lead to new insights into the complicated microcirculation phenomena such as the role of the glycocalyx on the blood flow behavior and the thrombogenesis process.

Because of their outstanding properties, rectangular PDMS microchannels are gaining popularity in biomedical research. In addition to providing the ability to investigate a variety of phenomena in the microcirculation (Takayama et al. 1999, Fujii 2002, Shevkoplyas et al. 2003), this kind of microfluidic device may be useful as a "lab-on-a-chip," a clinical diagnostic instrument. However, until now, precise measurements of blood flow behavior through this kind of microchannel has not been performed. In the present study, we demonstrated that by combining the rectangular PDMS microchannel with a confocal micro-PIV system it is possible to obtain both qualitative and quantitative information about the complex biophysical behavior of in vitro blood flow at a mesoscopic level. Thus, we believe that the present combination has potential as a powerful tool to investigate several phenomena in the microcirculation.

\section{Conclusions}

We applied a confocal micro-PIV system to examine PS and in vitro blood flowing in a rectangular PDMS microchannel with a low aspect ratio. For PS, the flattening of the velocity profiles was in good agreement with an established analytical solution for this kind of microfluidic device. However, when we assessed the flow of in vitro blood (20\% haematocrit), small fluctuations were observed in the velocity profiles at optically sectioned planes along the $z$-axis. Blood flow measurements performed at the centre plane of the microchannel clearly show small fluctuations on averaged ensemble velocity profiles. The reasons for the encountered "microturbulences" are not entirely clear, but our qualitative observations indicate that interactions between neighboring RBCs, the high shear rate generated in the vicinity of the walls, and temporal fluctuations in the haematocrit may play important roles in the hydrodynamic disturbance effects encountered in the blood velocity profiles. However, due to the variety of complex phenomena occurring at the microscale level, further studies are needed to clarify the presence of these fluctuations on the ensemble velocity profiles.

The measurements presented in this study clearly showed that confocal micro-PIV can be effectively integrated with a PDMS microchannel to obtain blood velocity profiles along the full depth of the microchannel because of its 3-D optical sectioning capability. This study also demonstrated that the combination of confocal micro-PIV systems with PDMS microchannels can play an important role in not only assessing existing theories, models, and computer simulations of the microcirculation, but also in the development of a more accurate biochip device for various biomedical applications, such as patient diagnosis and monitoring, drug delivery, and cancer cell detection. 


\section{Acknowledgements}

This study was supported in part by the following grants: International Doctoral Program in Engineering from the Ministry of Education, Culture, Sports, Science and Technology of Japan (MEXT), "Revolutionary Simulation Software (RSS21)" next-generation IT program of MEXT; Grants-in-Aid for Scientific Research from MEXT and JSPS Scientific Research in Priority Areas (768) "Biomechanics at Micro- and Nanoscale Levels," Scientific Research (A) No.16200031 "Mechanism of the formation, destruction, and movement of thrombi responsible for ischemia of vital organs". The authors also thank all members of Esashi, Ono and Tanaka Lab. for their assistance in fabricating the PDMS microchannel.

\section{References}

R. Adrian, Annu. Rev. Fluid Mech. 23 261-304 (1991).

C. Alonso, A. Pries, O. Kiesslich, D. Lerche and P. Gaehtgens Am. J. Physiol. 268 (1 Pt 2) H25-H32 (1995).

M. Baker and H. Wayland, H., Microvascular Research 7, 131-143 (1974).

D. Beebe, G. Mensing and G. Walker, Annu. Rev. Biomed. Eng. 4, 261-286 (2002).

L. Bitsch, L. Olesen, C. Westergaard, H. Bruus, H. Klank and J. Kutter, Experiments in Fluids 39 505-511 (2005).

J. Borenstein, H. Terai, K. King, E. Weinberg, M. Kaazempur-Mofrad and J. Vacanti, Biomedical Microdevices 4(3) $167-175$ (2002).

G. Born, A. Melling and J. Whitelaw, Biorheology 15, 163-172 (1978).

T. Brown, Journal of Biomechanics 33 3-14 (2000).

H. Bruus, Theoretical microfluidics (MIC, Technical University of Denmark, Denmark, 2004).

G. Bugliarello and J. Hayden, J., Trans. Soc. Rheol. 7, 209-230 (1963).

C. Caro,T. Pedley,R. Schroter and W. Seed, The mechanics of the circulation (Oxford University Press, 1978).

W. Chang, D. Akin, M. Sedlak, M. Ladisch and R. Bashir, Biomedical Microdevices 5(4) 281-290 (2003).

S. Chien, S. Usami and R. Skalak, Blood flow in small tubes Handbook of Physiology - The cardiovascular system IV (1984) 217-249.

T. Cochrane, J. Earnshaw and A. Love, Med. \& Biol. Eng. \& Comput. 19 589-596, (1981).

C. Duffy, J. McDonald, O. Schueller and G. Whitesides Anal. Chem. 70 4974-4984 (1998).

S. Einav, R. Berman, P. Fuhro, P. DiGiovanni, S. Fine and J. Fridman, Biorheology 12 207-210, (1975).

R. Fahraeus, T. Lindqvist, Am. J. Physiol. 96, 562-568 (1931).

M. Faivre, M. Abkarian, K. Bickraj and H. Stone, Biorheology 43 147-159, (2006).

T. Fujii, Microelectronic Engineering 61-62 907-914 (2002).

P. Gaehtgens, H. Meiselman and H. Wayland, Microvascular Research 2 13-23, (1970).

P. Gaehtgens, Biorheology 24 367-376 (1987).

S. Gifford, M. Frank,, J. Derganc, C. Gabel, R. Austin, T. Yoshida and W. Bitensky, Biophysical Journal 84 623-633 (2003).

H. Goldsmith and V. Turitto, Tromb. Haemost. 55 415-435 (1986).

H. Golster, M. Linden, S. Bertuglia, A. Colantuoni, G. Nilsson and F. Sjoberg, Microvascular research 58 62-73 (1999).

R. Gomez, R. Bashir, A. Sarikaya, M. Ladish, J. Sturgis, J. Robison, T. Geng, A. Bhunia, H. Apple and S. Wereley, , Biomedical Microdevices 3(3) 201-209 (2001).

G. Holzapfel, G. Sommer, C. Gasser, and P. Regitnig Am. J. Physiol Heart Circ. Physiol. 289 H2048-H2058 (2005).

H. Kaji, T. Kawashima and M. Nishizawa, Langmuir 22 10784-10787 (2006).

G. B. Kim and S. J. Lee, Experiments in Fluids 41 195-200 (2006).

H. Kinoshita, M. Oshima, S. Kaneda and T. Fujii, Proceedings of the $9^{\text {th }}$ ICMSCLS (Boston, Massachusetts, USA, 2005).

A. Koutsiaris, D. Mathioulakis and S. Tsangaris, Measurement Science and Technology 10 1037-1046 (1999).

R. Lima, Analysis of the blood flow behavior through microchannels by confocal micro-PIV/PTV system, Doctoral Thesis (2007), Tohoku University, Japan.

R. Lima, S. Wada, K. Tsubota and T. Yamaguchi, Proceedings of the $3^{\text {rd }}$ IASTED ICB - BioMech (Benidorm, Spain, 485, 2005).

R. Lima, S. Wada, K. Tsubota and T. Yamaguchi, Measurement Science and Technology 17, 797-808 (2006).

R. Lima, S. Wada, M. Takeda, K. Tsubota and T. Yamaguchi, Journal of Biomechanics 40, 2752-2757 (2007).

N. Maeda, Japanese Journal of Physiology 46 1-14, (1996).

A. Mata, A. Fleischman and S. Roy, Biomedical Microdevices 7:4 281-293 (2005).

J. McDonald and G. Whitesides, Accounts of Chemical Research 35 (7) 491-499 (2002).

G. Mchedlishvili and N. Maeda, Japanese Journal of Physiology 51 19-30, (2001).

C. Meinhart, S. Wereley and J. Santiago, Journal of Fluids Engineering 122 285-289 (2000).

G. Minas, J. Martins, J. Ribeiro, R. Wolffenbuttel and J. Correia, Sensors and Actuators 110 33-38 (2004). 
J. Moger, S. Matcher, C. Winlove and A. Shore, Journal of Biomedical Optics 9(5) 982-994, (2004).

N. Mortensen, F. Okkels and H. Bruus, Physical Review E 71 1-4 (2005).

A. Nakano, Y. Sugii, M. Minamiyama and H. Niimi, Clinical Hemorheology and Microcirculation $29445-$ 455(2003).

N. Nguyen and S. Wereley, Fundamentals and applications of microfluidics (Norwood, MA: Artech House, Inc. , 2002).

J. Park, C. Choi and K. Kihm, Experiments in Fluids 37 105-119 (2004).

J. Park and K. Kihm, Optics and Lasers in Engineering 44, 208-223 (2006).

A. Parthasarathi, S. Japee and R. Pittman, Annals of Biomedical Engineering 27 313-325. (1999).

A. Pries, T. Secomb, T. Gessner, M. Sperandio, J. Gross and P. Gaehtgens, Circulation Research 75 904-915 (1994).

A. Pries and T. Secomb, Clinical Hemorheology and Microcirculation 29 143-148 (2003).

M. Raffel, C. Willert and J. Kompenhans, Particle image velocimetry: a practical guide (Springer-Verlag, Germany, 1998).

Rika nenpyo, Chronological scientific tables, (National Astronomical Observatory), Maruzen Co., Japan, 1996.

J. Santiago, S. Wereley, C. Meinhart, D. Beebe and R. Adrian, Experiments in Fluids 25 316-319 (1998).

T. Secomb, Symp. Soc. Exp. Biol. (London, UK, 49 305-321, 1995).

S. Shevkoplyas, S. Gifford, T. Yoshida and M. Bitensky, Microvascular Research 65 132-136 (2003).

M. Shin, K. Matsuda, O. Ishii, H. Terai, M. Kaazempur-Mofrad, J. Borenstein, M. Detmar and J. Vacanti, Biomedical Microdevices 6:4 269-278 (2004).

H. Steiger, R. Aaslid, S. Keller and H. Reulen, Heart and Vessels, 5 (1) 41-46 (1989).

N. Sutton, M. Tracey, I. Johnston, R. Greenaway and M. Rampling, Microvascular Research 53 272-281(1997).

Y. Suzuki, N. Tateishi, M. Soutani and N. Maeda, Microcirculation 3 49-57, (1996).

P. Tabeling, Proceedings of the $14^{\text {th }}$ Australasian Fluid Mechanics Conference (Adelaide, Autralia, 2001).

S. Takayama, J. McDonald, E. Ostuni, M. Liang, P. Kenis, F. Ismagilov and G. Whitesides Patterning cells and their environments using multiple laminar fluid flows in capillary networks Proc. Natl. Acad. Sci. 96, 5545-5548(1999).

G. Tangelder, D. Slaaf, M. Muijtjens, T. Arts, M. Egbrink and R. Reneman, Circulation Research 59 505-514 (1986).

T. Tanaani, S. Otsuki, N. Tomosada, Y. Kosugi, M. Shimizu and H. Ishida, Applied Optics 4122 4704-4708 (2002).

T. Thorsen, S. Maerkl and S. Quake, Science, 298 580-584 (2002).

M. Toner and D. Irimia, Annu. Rev. Biomed. Eng. 7, 77-103 (2005).

K. Tsukada, H.Minamitani, E. Sekizuka, C. Oshio, Physiol Meas. 21(4) 459-471 (2000).

W. Uijttewaal, E. Nijhof and R. Heethaar, Journal of Biomechanics 27 35-42 (1994).

M. Unger, H. Chou, T. Thorsen, A. Scherer and S. Quake, Science, 288 113-116 (2000).

P. Vennemann, K. Kiger, R. Lindken, B. Groenendijk, S. Stekelenburg-de Vos, T. Hagen, N. Ursem, R. Poelmann, J. Westerweel and B. Hierk, Journal of Biomechanics 39 1191-1200 (2006).

S. Wilhelm, B. Grobler, M. Gluch and H. Heinz Confocal laser scanning microscopy: principles (Carl Zeiss, Germany, 2003)

C. Willert, M. Raffel, J. Kompenhans, B. Stasicki and C. Kahler, Flow Meas. Instrum. 7 247-256 (1996). 